\title{
Heart Failure: Insights into Device Therapy
}

\author{
Md. Abdul Kader Akanda \\ Prof \& Head, Department of Cardiology, Sir Salimullah Medical College and Mitford Hospital, \\ President, Heart Failure Research Foundation.
}

(Cardiovasc j 2021; 14(1): 1-3)

Although pharmacologic therapy has made impressive advances in the past decade and is the mainstay of therapy for heart failure (HF), there is still a large unmet need, because morbidity and mortality remain unacceptably high. Implanted medical devices are gaining increasing utility in this group of patients and have the potentials to revolutionize the treatment of heart failure. The majority of devices in clinical use or under active investigation in $\mathrm{HF}$ can be grouped into 1 of 4 categories: devices to monitor HF condition, devices to treat rhythm disturbances, devices to improve the mechanical efficacy of the heart and devices to replace part or all of the hearts function. ${ }^{1}$ The management of HF has changed significantly over the last 30 years, leading improvements in the quality of life and outcomes, at least for patients with a substantially reduced left ventricular ejection fraction (HFrEF). Device therapy in $\mathrm{HF}$ includes the implantable cardioverter defibrillator (ICD) and Cardiac resynchronization therapy (CRT). Beyond improving the quality of life and reducing the morbidity, CRT has also shown mortality benefit in selected patients. ${ }^{2}$

Chronic heart failure with reduced EF:

Use of cardiac intervention in chronic HF is mostly limited to implantation of ICD for primary or secondary prevention of sudden cardiac death (SCD) and implantation of CRT according to the indications as directed by guidelines of ESC, ACC/AHA etc. ICD reduces the risk of SCD and all causes mortality. CRT improves cardiac function and also improves HF symptoms and QOL (Quality of life) by restoring synchronous contraction of heart. The COMPANION trial and CARE-HF trials have showed superiority of CRT and CRT-D over optimal medical therapy in selected $\mathrm{HF}$ patients, in reducing mortality and $\mathrm{HF}$ related hospitalization. ${ }^{3,4}$

\section{Cardiac contractility modulation:}

Chronic HF patients that remain symptomatic with medical management or cannot tolerate maximum medical therapy and do not fulfil the criteria for CRT implantation, require new approach of treatment or new devices. CCM is a newer form of implantable device approved by FDA in March' 2019 for $\mathrm{HFrEF}$ patients who remain symptomatic despite medical therapy and not a candidate of CRT. It gives non excitatory electrical stimulation to ventricles during the absolute refractory period to enhance cardiac contractility without activating extra systolic contraction. It is being evaluated in patients with HFrEF who are in NYHA III or IV and found to improved exercise tolerance ( $\uparrow$ peak Vo2), 6 min walk distance and QOL (Quality of life) ${ }^{5}$.

\section{Percutaneous ventricular restoration therapy:}

Restoration of LV geometry and function is being considered by isolating damaged and nonfunctional muscle segments from functional myocardium to decrease the $L V$ volume for patients with ischemic HF. It is done by trans catheter implantation of cardiokinetix parachute device. PARACHUTE IV trial is a multi-center RCT which is going on in different centers of USA \& Canada evaluating the efficacy of this device on outcomes for patients with ischemic HF. 6,7

\section{For heart failure with preserve EF LA Decompression:}

Left $\mathrm{HF}$ is associated with resting or exercise induced increase in LAP and pulmonary

Address of Correspondence: Prof. Md. Abdul Kader Akanda, Prof \& Head, Department of Cardiology, Sir Salimullah Medical College and Mitford Hospital, President, Heart Failure Research Foundation.

๑ 2020 author; licensed and published by International Society of Cardiovascular Ultrasound, Bangladesh Chapter and Bangladesh Society of Geriatric Cardiology. This is an Open Access article distributed under the terms of the CC BY NC 4.0 (https://creativecommons.org/licenses/by-nc/4.0). 
congestion. There is also volume overload in $\mathrm{HF}$ patients, especially in HFrEF group. Medical management of $\mathrm{HF}$ is mainly aimed at reducing total body volume and intravascular pressure, especially in HFrEF but not in HFpEF. For the later group of patients, reducing LAP may confer symptomatic benefits. Scientists have developed devices to make communication between LA and RA and these reducing LAP. ${ }^{8}$

Three device systems are now available for maintaining the inter-atrial communications such as IASD system, V-wave device and AFR (Atrial flow regulator). IASD and AFR maintains bidirectional flow and $\mathrm{V}$-wave device maintain unidirectional flow from LA to RA. These devices are deployed after trans-septal puncture. This device usually creates a shunt where Qp: Qs remains $<1.3$. So, $R A$ and $R V$ can withstand with the extra load from LA for decades without compromising RV function. REDUCE - LAP - HF trial, phase I have evaluated IASD system, corvia medical, in patient of $\mathrm{HFpEF}$ and $\mathrm{HFmrEF}$ $(\mathrm{EF} \geq 40 \%)$. This study revealed $97 \%$ implantation success, 95\% 1-year survival, improved NYHA class $(\mathrm{P} \leq 0.001)$, improved $6 \mathrm{~min}$ walking distance by $33 \mathrm{~m}$ $(\mathrm{P} \leq 0.001)$, significant fall in LVEDP $(\mathrm{P} \leq 0.001)$, significant rise in $\mathrm{RVEF}(\mathrm{P} \leq 0.001)$ and reduced hospitalization $(\mathrm{P} \leq 0.05)$. Based on this study IASD system received $\mathrm{CE}$ mark approval for patients with $\mathrm{HF}$ with $\mathrm{EF} \geq 40 \% .{ }^{9,10}$ A large scale trial REDUCELAP-HF II, is going on at this moment. ${ }^{11}$

' $\mathrm{V}$ ' wave atrial septal shunt device has received break through status by FDA following a small clinical trial on 16 patients. ${ }^{12} \mathrm{~A}$ large is under way called "RELLEVE-HF" trial which would include 500 patients. ${ }^{13}$ Atrial flow regulator has also received breakthrough status by FDA following AFR-PRELIVE trial. All of these inter-atrial shunt devices are in their early stages of evaluation and large studies would reveal their safety and efficacy. ${ }^{14,15}$

\section{Implantable Heart Failure monitor:}

It is one of the important advancements by scientists for HF patients. Currently St. Judes Cardio MEMS HF system is the only FDA approved device which is inserted into pulmonary artery (PA) via a trans catheter approach. It detects the PA pressure and monitor the fluid retention due to worsening HF symptoms. Adjustments of medications can be made according to the pressure parameters and thus HF related hospitalization and expenditure can be reduced. ${ }^{16,17}$

\section{Mechanical circulatory support in right heart failure (MCS):}

Mechanical circulatory support is reserved for patient refractory to optimal management of acute or chronic right heart failure, to bridge to recovery, to bridge to heart, lung or heart lung transplantation and as destination therapy (permanent use). $42 \%$ to $75 \%$ may recover to allow MCS device explantation in acute form of heart failure.

In spite of its substantial benefits, CRT is underutilized even in developed countries like US $\&$ Europe. The number of CRT implants increased from 13,000 to 55,000 per year from 2002 to 2005 in USA: after 2005 the number of implants did not increase. ${ }^{18}$ Hence even the developed nation citizens are finding it difficult to adopt such a promising but costly therapy. The situation is obviously worse in developing countries in the background of a strained healthcare economy. The cost burden it imparts to a developing economy remains its Achilles heel. For cost consideration we often only think about the first implantation; the cost of delayed complications and generator replacement should also be considered. The main hindrances for optimal use of cardiac implantable electronic devices (CIED) in HF patients include cost, understanding of the disease from patient and physician perspective, risk involved with implantation procedure, availability of experienced implanters in many areas, lack of dedicated HF clinic for specialized management of $\mathrm{HF}$ and need for long term follow up and management. ${ }^{2}$

Device therapy is a boon for HF patients but should be considered only after exhausting aggressive GDMT. Since the patients of developing countries like Bangladesh spend out-of-pocket for their health, the available therapies are unaffordable to the vast majority of the population. So, there is an urgent need to intervene to make the therapy affordable and available to $\mathrm{HF}$ patients. Development of national guideline for device therapy and follow up protocol according to national economy, development of trained manpower for device implantation and dedicated heart failure centres to ensure specialized service and research 
work to give insights into the ethnic variation of management.

\section{Conflict of Interest - None.}

\section{References:}

1. Boehmer J. Device therapy for heart failure. Am $J$ Cardiol. 2003; 91(6): 53-59. DOI:10.1016/s00029149(02)03380-5

2. Mishra S, Mohan J, Nair T et al. Management protocols for chronic heart failure in India. Indian Heart J. 2018; 70(1): 105-127. DOI:10.1016/j.ihj.2017.11.015

3. Bristow M, Saxon L, Boehmer J et al. CardiacResynchronization Therapy with or without an Implantable Defibrillator in Advanced Chronic Heart Failure. $N$ Engl J Med. 2004; 350(21): 2140-2150. DOI:10.1056/nejmoa032423

4. Cleland J, Daubert J, Erdmann E et al. The Effect of Cardiac Resynchronization on Morbidity and Mortality in Heart Failure. $N$ Engl J Med. 2005; 352(15): 15391549. DOI:10.1056/nejmoa050496

5. Ponikowski P, Voors A, Anker S et al. 2016 ESC Guidelines for the diagnosis and treatment of acute and chronic heart failure. Eur Heart J. 2016; 37(27): 2129-2200. DOI:10.1093/eurheartj/ehw128

6. Thomas M, Nienaber $\mathrm{C}$, Ince $\mathrm{H}$ et al. Percutaneous ventricular restoration (PVR) therapy using the Parachute device in 100 subjects with ischaemic dilated heart failure: one-year primary endpoint results of PARACHUTE III, a European trial. EuroIntervention. 2015; 11(6): 710-717. DOI:10.4244/eijv11i6a143

7. Costa M, Mazzaferri E, Sievert H et al. Percutaneous Ventricular Restoration Using the Parachute Device in Patients With Ischemic Heart Failure. Circulation: Heart Failure. 2014; 7(5): 752-758. DOI:10.1161/circheartfailure. 114.001127

8. Søndergaard L, Reddy V, Kaye D et al. Transcatheter treatment of heart failure with preserved or mildly reduced ejection fraction using a novel interatrial implant to lower left atrial pressure. Eur J Heart Fail. 2014; 16(7): 796-801. DOI:10.1002/ejhf.111

9. Hasenfuß G, Hayward C, Burkhoff D et al. A transcatheter intracardiac shunt device for heart failure with preserved ejection fraction (REDUCE LAP-HF): a multicentre, open-label, single-arm, phase 1 trial. The Lancet. 2016; 387(10025): 1298-1304. DOI:10.1016/ s0140-6736(16)00704-2

10. Kaye D, Hasenfuß G, Neuzil P et al. One-Year Outcomes After Transcatheter Insertion of an Interatrial Shunt Device for the Management of Heart Failure With Preserved Ejection Fraction. Circulation: Heart Failure. 2016; 9(12). DOI:10.1161/circheartfailure. 116.003662

11. Berry N, Mauri L, Feldman T et al. Transcatheter InterAtrial Shunt Device for the treatment of heart failure: Rationale and design of the pivotal randomized trial to REDUCE Elevated Left Atrial Pressure in Patients with Heart Failure II (REDUCE LAP-HF II). Am Heart J. 2020; 226: 222-231. DOI:10.1016/ j.ahj.2019.10.015

12. Lilly SM, Burkhoff D. Technologies for Treating Left Atrial Decompression in Heart Failure. Cardiac Interventions Today. 2018; 12(1): 59-97.

13. Reducing Lung CongestIon Symptoms in Advanced Heart Failure - Full Text View - ClinicalTrials.gov. Clinicaltrials.gov. https://clinicaltrials.gov/ct2/show/ NCT03499236. Published 2021. Accessed July 9, 2021.

14. Paitazoglou C, Özdemir R, Pfister R et al. The AFRPRELIEVE trial: a prospective, non-randomised, pilot study to assess the Atrial Flow Regulator (AFR) in heart failure patients with either preserved or reduced ejection fraction. EuroIntervention. 2019; 15(5): 403410. DOI:10.4244/eij-d-19-00342

15. Patel M, Samuel B, Girgis R, Parlmer M, Vettukattil J. Implantable atrial flow regulator for severe, irreversible pulmonary arterial hypertension. EuroIntervention. 2015; 11(6): 706-709. DOI:10.4244/eijy15m07_08

16. Abraham W, Adamson P, Bourge R et al. Wireless pulmonary artery haemodynamic monitoring in chronic heart failure: a randomised controlled trial. The Lancet. 2011; 377(9766): 658-666. DOI:10.1016/s01406736(11)60101-3

17. Jermyn R, Alam A, Kvasic J, Saeed O, Jorde U. Hemodynamic-guided heart-failure management using a wireless implantable sensor: Infrastructure, methods, and results in a community heart failure diseasemanagement program. Clin Cardiol. 2016; 40(3): 170176. DOI:10.1002/clc. 22643

18. Bank A, Gage R, Olshansky B. On the Underutilization of Cardiac Resynchronization Therapy. J Card Fail. 2014; 20(9): 696-705. DOI:10.1016/j.cardfail.2014.06.005 